\title{
Nonlinear mixing behavior of the three-dimensional Rayleigh-Taylor instability at a decelerating interface ${ }^{\text {a) }}$
}

\author{
R. P. Drake, ${ }^{\text {b) }}$ D. R. Leibrandt, E. C. Harding, C. C. Kuranz, and M. A. Blackburn \\ University of Michigan, Atmospheric Oceanic and Space Sciences, 2455 Hayward Street, Ann Arbor, \\ Michigan 48109-2143 \\ H. F. Robey, B. A. Remington, M. J. Edwards, A. R. Miles, T. S. Perry, \\ R. J. Wallace, and H. Louis \\ Lawrence Livermore National Laboratory, P.O. Box 808, Livermore, California 94550 \\ J. P. Knauer \\ University of Rochester, 250 East River Road, Rochester, New York 14623 \\ D. Arnett \\ University of Arizona, 5333 N. Camino Real, Tucson, Arizona 85718
}

(Received 31 October 2003; accepted 6 January 2004; published online 23 April 2004)

\begin{abstract}
Results are reported from the first experiments to explore the evolution of the Rayleigh-Taylor (RT) instability from intentionally three-dimensional (3D) initial conditions at an embedded, decelerating interface in a high-Reynolds-number flow. The experiments used $\sim 5 \mathrm{~kJ}$ of laser energy to produce a blast wave in polyimide and/or brominated plastic having an initial pressure of $\sim 50$ Mbars. This blast wave shocked and then decelerated the perturbed interface between the first material and lower-density $\mathrm{C}$ foam. This caused the formation of a decelerating interface with an Atwood number $\sim 2 / 3$, producing a long-term positive growth rate for the RT instability. The initial perturbations were a 3D perturbation in an "egg-crate" pattern with feature spacings of $71 \mu \mathrm{m}$ in two orthogonal directions and peak-to-valley amplitudes of $5 \mu \mathrm{m}$. The resulting RT spikes appear to overtake the shock waves, moving at a large fraction of the predeceleration, "free-fall" velocity. This result was unanticipated by prior simulations and models. (C) 2004 American Institute of Physics.
\end{abstract}

[DOI: $10.1063 / 1.1651492]$

\section{INTRODUCTION}

The Rayleigh-Taylor (RT) instability ${ }^{1,2}$ is a ubiquitous phenomenon, having consequences in the dynamics of atmospheric circulation, in laboratory fluids and gasses, in magnetized plasmas from those produced for fusion to planetary magnetospheres, and in supernovae, to name only a few. This ubiquity has made RT a subject of extensive study, leading to hundreds of published papers in the last decade alone. It is not surprising that there are many specific regimes of RT, corresponding to specific aspects of various physical systems such as surface tension or granularity. Our focus here is on experiments exploring the behavior of the RT instability, developing from initial conditions with three-dimensional (3D) structure, at interfaces that are shocked and then decelerated by blast waves of high Mach number, in compressible flows of very high Reynolds number. This is the regime of interest to astrophysical phenomena such as supernovae, and accessible in the laboratory only by high-energy-density experimental techniques. The experiments we report were motivated by the desire to observe the evolution of RT from initial conditions with 3D structure, including the transition to broadband fluid turbulence. We have observed the devel-

\footnotetext{
${ }^{a)}$ Paper CI1 4, Bull. Am. Phys. Soc. 48, 48 (2003).

${ }^{b)}$ Invited speaker.
}

opment of complex 3D structures that transport denser material through less dense material faster than a standard buoyancy-drag model predicts.

In all environments, the RT instability causes regions of less dense, buoyant material, known as "bubbles," to float upward, and regions of more dense, "heavy" material, known as "spikes," to plunge downward. Both of these structures experience drag, which causes the "bubble tips" and "spike tips" at their ends to broaden, often followed by Kelvin-Helmholtz instabilities. There has been partial success at modeling RT using models based on the competition between buoyancy and drag. The vast majority of work on RT has explored the development of the instability from twodimensional (2D) initial conditions, such as the sinusoidal modulation of an interface. This is the most straightforward situation to produce, to diagnose, and to simulate. In the realm of high-energy-density systems, this has included many studies of RT behavior at an ablation surface, ${ }^{3-5}$ motivated by inertial fusion, and a number of studies ${ }^{6-14}$ of RT behavior at a decelerating, embedded interface, motivated by basic science and/or astrophysics. In recent work with decelerating interfaces, buoyancy-drag models have proven successful, after adjusting for compressibility effects, in explaining observations with 2D, single-mode perturbations, while 2D simulations have proven able to reproduce, on the whole, observations with 2D multimode perturbations. ${ }^{15}$

In addition, simulations of complex astrophysical phe- 


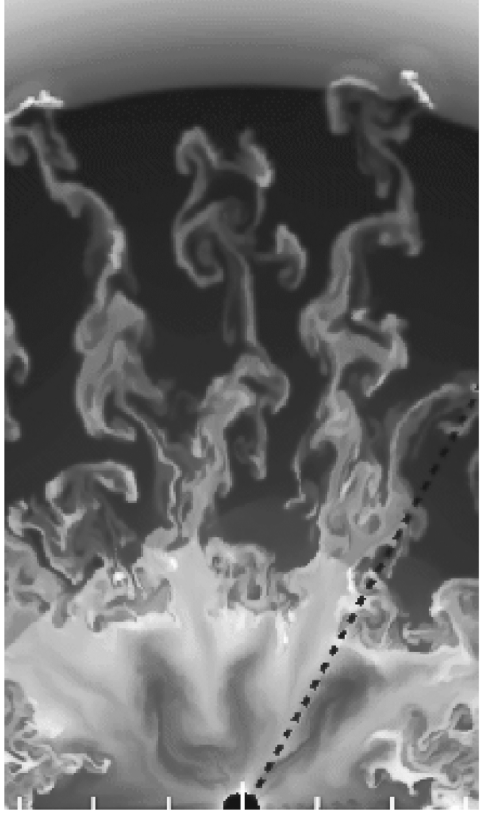

FIG. 1. Density structures in a two-dimensional simulation of SN 1987A during its explosion. Used with permission from K. Kifonidis.

nomena remain largely 2D. Thus, for example, Fig. 1 shows results of a 2D simulation of the explosion of SN 1987A (Kifonidis, Max Planck Garching, private communication), obtained as part of a study of the role of neutrino-driven convection in this explosion. ${ }^{16}$ One sees well-developed RT spikes of material from the interior of the star, penetrating through the He layer toward the $\mathrm{He}-\mathrm{H}$ interface. The shear along the edges of these RT spikes drives the KelvinHelmholtz instability, which produces the observed rollups. One significant question in the context of these simulations is how RT would differ in 3D, and in particular how quickly it might deliver matter from the interior to the $\mathrm{He}-\mathrm{H}$ interface. This happens later in the work of Kifonidis than in some earlier 2D simulations. ${ }^{17}$ The timing matters because it determines whether the interior matter penetrates far beyond the $\mathrm{He}-\mathrm{H}$ interface or gets stuck behind it for at least some time. Only the former would be consistent with the observations (on the assumption of a globally symmetric explosion).

In the literature, there is only limited work on RT in 3D, high-Re systems. The simulations have either been focused on the behavior of isolated bubbles or spikes ${ }^{18-21}$ or on multimode phenomena in thin, ablative layers relevant to inertial fusion. ${ }^{22}$ There is, to our knowledge, only one exploration by detailed simulations in 3D of multimode RT at an embedded interface, ${ }^{23}$ but it is a calculation for incompressible fluids at low Reynolds number, Re. Earlier work with 3D simulations includes low-resolution, multimode simulations by Youngs, ${ }^{24}$ work with a modal model in $3 \mathrm{D},{ }^{25}$ and preliminary $3 \mathrm{D}$ calculations of growth from random perturbations. ${ }^{21} \mathrm{We}$ are aware of no work to date addressing the impact of varying initial conditions on RT in 3D. The only 3D experiments were focused on RT at an ablation layer, ${ }^{26,27}$ again motivated by inertial fusion. In contrast, the $3 \mathrm{D}$ experimental work in low-Re, low-Mach-number systems is too vast to cite. We

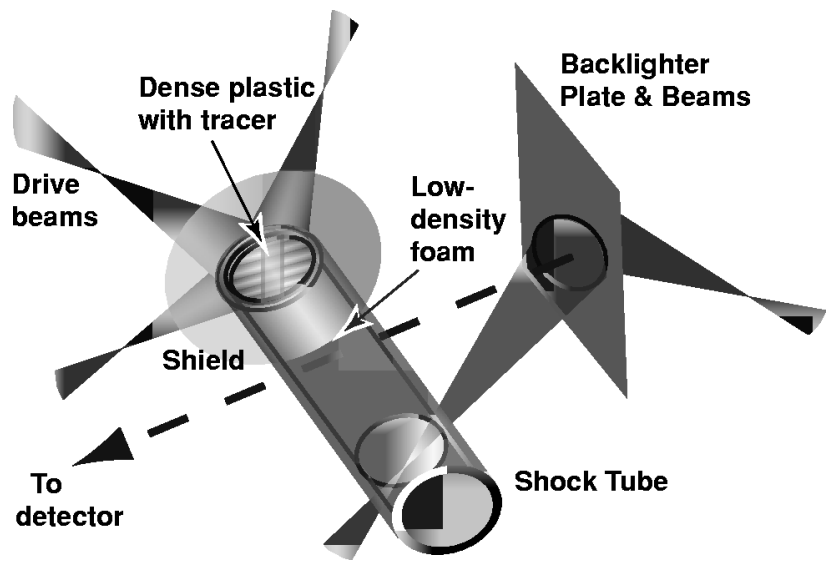

FIG. 2. Target geometry for experiments.

mention here only that of Dimonte and Schneider, ${ }^{28}$ which has had a significant impact on recent theoretical work.

\section{EXPERIMENTAL CONFIGURATION AND CONDITIONS}

\section{A. Target structure}

Figure 2 is a drawing of the target used for these experiments. The drive lasers irradiate a polyimide surface, 800 $\mu \mathrm{m}$ in diameter. The polyimide used has chemical structure $\mathrm{C}_{22} \mathrm{H}_{10} \mathrm{O}_{5} \mathrm{~N}_{2}$ and a density of $1.41 \mathrm{~g} / \mathrm{cm}^{3}$. It is $150 \mu \mathrm{m}$ thick. At the rear surface, a $200-\mu \mathrm{m}$-wide, 75 - $\mu \mathrm{m}$-deep slot has been milled in the polyimide. A strip of $\mathrm{C}_{500} \mathrm{H}_{457} \mathrm{Br}_{43}$, of density $1.42 \mathrm{~g} / \mathrm{cm}^{3}$, has been glued into the slot. These two materials were chosen to have nearly the same density and to be dominantly low- $Z$, so that their hydrodynamic response to pressures of tens of Mbars would be similar. The role of the strip is to provide a thin layer that more strongly absorbs the $\mathrm{X}$ rays used to radiograph the target during the experiment, so that one can observe the structure near the center of the surface without obscuration by edge effects. In some targets, which we will refer to as "modulated targets," the rear surface of this assembly was then machined to produce the eggcrate structure shown in Fig. 3. The amplitude of the pattern defined by the machining tool is $a_{0} \sin \left(k_{x} x\right) \sin \left(k_{y} y\right)$, with $a_{0}$ $=2.5 \mu \mathrm{m}$ and $k_{x}=k_{y}=2 \pi /(71 \mu \mathrm{m})$. One can see in the figure that tearing of the material during machining produced substantial additional structure of smaller amplitude and shorter wavelength. The potential significance of this is dis-

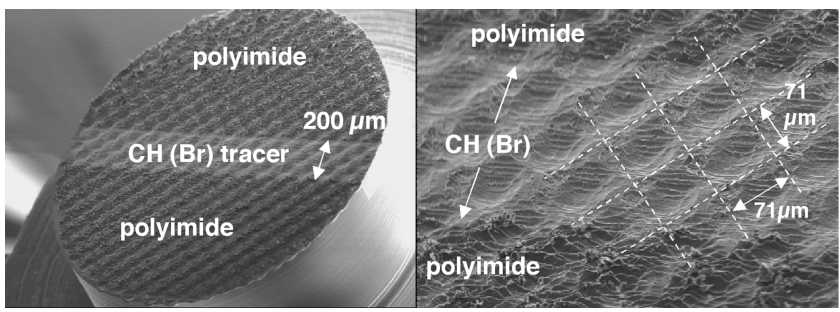

FIG. 3. Images of the machined, perturbed surface of a dense layer in a target. (a) Overview, (b) closeup showing mode structure and small-scale features. 
cussed below. We will refer to those targets with nominally planar plastic-foam interfaces as "planar targets."

Following the initial plastic materials was an $\sim 2.4$ - mm-long cylinder of carbon foam, again $800 \mu \mathrm{m}$ in diameter. The foam is carbonized resorcinol formaldehyde. It has an open cell structure, with characteristic cell sizes of $<0.1 \mu \mathrm{m}$. The surfaces of the ends of the foam were planar, and were smoothed so that the surface finish is better than 1 $\mu \mathrm{m}$. Foam densities of $50 \mathrm{mg} / \mathrm{cm}^{3}$ and $100 \mathrm{mg} / \mathrm{cm}^{3}$ were used in various experiments. Data from both densities are shown in the figures, as indicated. The experimental results in the two cases were qualitatively identical.

A Be shock tube of $1100 \mu \mathrm{m}$ outside diameter and 800 $\mu \mathrm{m}$ inside diameter held the cylindrical structures just described, and the driven end of the target included a shield of $2.5 \mathrm{~mm}$ diam to protect the outer target structures from laser irradiation.

\section{B. Laser irradiation and target diagnostics}

Ten beams of the Omega ${ }^{29}$ laser irradiate the polyimide surface of the target at a wavelength of $0.35 \mu \mathrm{m}$. The laser pulses are of $1 \mathrm{~ns}$ full width at half maximum, with approximately 100-ps rise and fall times and approximately flat tops. The energy in each laser beam is typically $450 \mathrm{~J}$ $( \pm 10 \%)$. Each beam passes through a distributed phase plate, producing a spot with a smooth overall profile and fine speckles on a 5- $\mu \mathrm{m}$ scale. The combined intensity profile of the 10 beams has a broad maximum and has fallen off about $10 \%$ at the $800-\mu \mathrm{m}$ diameter of the target, so that the average irradiance is $9 \times 10^{14} \mathrm{~W} / \mathrm{cm}^{2}$. This irradiation produces an ablation pressure of approximately 50 Mbars (based on simulations that match the velocity of the shock wave produced). Because shock waves anneal rapidly, the fine-scale modulations in the irradiance will not produce structure in the shock when it reaches the embedded interface.

The role of preheat in these experiments remains incompletely explored, and is a topic for future research. The laser irradiation produces both $\mathrm{x}$ rays and electrons that penetrate the target and heat the interface before it is reached by the shock. The x-ray preheat is calculated, in the simulations described below, to heat the interface to a temperature of 0.3 $\mathrm{eV}$ and produce about $2 \mu \mathrm{m}$ of motion before the shock arrives. The amount and spectrum of the energetic electrons is not well known. They could potentially have a larger effect, but experiments will be needed to quantify this. The essential impact of any preheat is to alter the initial conditions that are encountered by the shock. This could certainly affect the detailed structures present in the deep nonlinear regime, but seems unlikely to have a dominant effect on the onset of turbulence or the overall spike penetration.

The principal diagnostic of these experiments is x-ray radiography. Several additional laser beams irradiate both sides of a thin metal target, typically Sc, located approximately $4 \mathrm{~mm}$ from the shock tube, as is shown in Fig. 2. The properties of these beams are nearly identical to those of the drive beams, although they are at times focused without phase plates to a somewhat smaller spot. These backlighter beams are delayed by 10 to 30 ns relative to the drive beam, so that they can detect the interface after it has evolved over some distance. The $\mathrm{x}$-ray radiation at several $\mathrm{keV}$, which is dominated by the $K \alpha$ radiation (at $4.3 \mathrm{keV}$ for Sc), passes through the target, is imaged by an array of 16 pinholes, and is detected by a gated detector. ${ }^{30}$ The 16 images are offset slightly in time, being distributed within the 1-ns backlighter pulse, but the evolution is gradual enough that they are effectively simultaneous. A gold grid, mounted on the side of the target, provides a calibration of both location and magnification.

\section{Experimental conditions}

Given the limited ability to diagnose these dense plasmas, we are forced to rely on simulations for an assessment of some experimental parameters. For this purpose, we used the HYADES code, ${ }^{31}$ a one-dimensional (1D), Lagrangian, single-fluid, three-temperature code with multigroup diffusive radiation transport. It is well known (D. G. Braun, Lawrence Livermore Laboratory, private communication) that such 1D simulations, run with the actual laser intensity, produce too large an ablation pressure as a consequence of their ignorance of lateral heat transport. By comparison with previous experiments, we determined that using an irradiance of $4.2 \times 10^{14} \mathrm{~W} / \mathrm{cm}^{2}$ in the simulation (a bit less than half the experimental irradiance) produces shock and interface motions that are generally consistent with observations.

The shock wave produced by the laser propagates through half the dense plastic (the polyimide layer) before the laser pulse ends, after which the front-surface rarefaction overtakes the shock to form a blast wave before the shock reaches the plastic-foam interface. This produces the desired blast-wave structure, with an abrupt acceleration by the shock followed by an extended deceleration in the rarefaction. When the blast wave reaches the interface, the dense plasma in the plastic rarefies, launching a shock into the foam, initially at $\sim 100 \mathrm{~km} / \mathrm{s}$. The velocity of the interface is $\sim 70 \mathrm{~km} / \mathrm{s}$ at first, decreasing to $\sim 40 \mathrm{~km} / \mathrm{s}$ by $20 \mathrm{~ns}$. This deceleration creates a positive growth rate for the RayleighTaylor instability. Figure 4 shows results from a 1D simulation. This simulation reproduces the observed timing and velocity of the shock wave in the foam until $17 \mathrm{~ns}$; at later times, the simulated shock is faster than the observed one. At $17 \mathrm{~ns}$, the peak pressure produced by the shock wave has been reduced to $\sim 1 \mathrm{Mbar}$, and the post-shock temperature in the plasma is $\sim 15 \mathrm{eV}$. The shock velocity is $60 \mathrm{~km} / \mathrm{s}$.

One can see in Fig. 4 the typical structure that develops when a blast wave crosses an interface at which the density decreases. A forward shock propagates into the low-density material, producing compression, acceleration, and heating. We will refer to this as the "shock" in the foam. In detail, the decrease in density, velocity, and pressure behind this shock is characteristic of decelerating shock waves. (The profiles would be flat in a steady shock wave.) The interface with the plastic, at $\sim 950 \mu \mathrm{m}$ at $17 \mathrm{~ns}$, is quite evident in the density and temperature. Further to the left is the reverse shock, at $\sim 600 \mu \mathrm{m}$ at $13 \mathrm{~ns}$. This is a weak shock, not steady in time. The density and pressure of the material between the two shocks decrease gradually with time, after the expansion of 


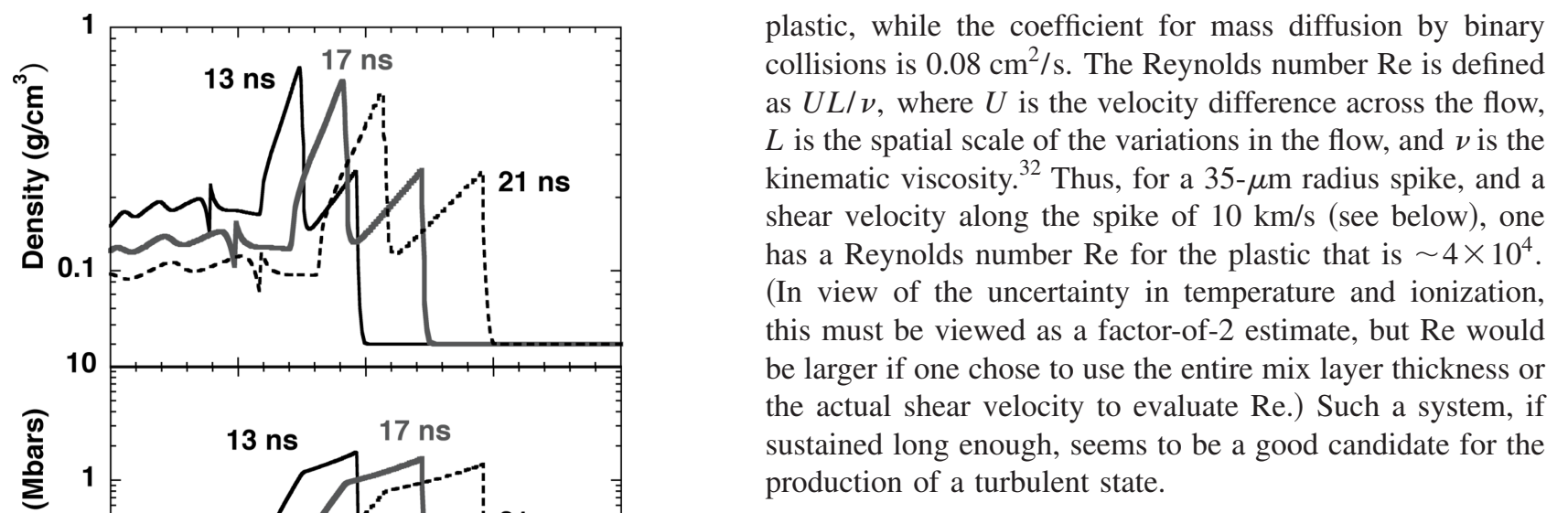

\section{EXPERIMENTAL RESULTS}

The principal data from these experiments were radiographic images obtained at selected times. The framing camera produced up to 16 images separated in time by a total of 800 ps. In most cases, the signal-to-noise ratio of these images could be improved by adding them, in which case the fluid motion at $\sim 45 \mathrm{~km} / \mathrm{s}$ produces a horizontal smearing of $36 \mu \mathrm{m}$. In theory, this could degrade the resolution of the image from the fundamental limit of $10 \mu \mathrm{m}$ due to the pinhole. In practice, the noise from local gain variations in the framing camera combined with the limited x-ray flux precludes one from attaining this resolution, so that averaging the images often produces better results. In the images, the spatial scale is defined so that the front surface of the target is at $0 \mu \mathrm{m}$ in $x$ and the axis of the target is at $0 \mu \mathrm{m}$ in $y$. The foam material thus begins at $150 \mu \mathrm{m}$.

Figures 5(a)-5(f) show a set of radiographic images. The images are in pairs, with an image from a modulated target presented above an image, taken at the same time, from an experiment with a planar target. We were able to obtain one such image in each individual laser experiment. However, we were unable to obtain enough data to determine the reproducibility of the fine details at any specific time.

Some experimental details deserve mention. The color scale on these images has been adjusted to provide the best possible view of the structures near the interface. Other features, such as the fiducial grid in the upper right corners and the dense material between the reverse shock and the interface, are resolved in the data though not very visible in this presentation of it. The fiducial grid location was measured before the experiment and used to calibrate the magnification and location of the image. The images are recorded on active strips of limited width. This produces the dark areas where there is no signal at the lower left and upper right of the images shown. In addition, a flaw in the detector on one image produces the dark dot on the images, and clipping of some images can produce brightness changes in the added images.

Next consider the physical features seen in these images. There is a bright region toward the left. This is the lowdensity material flowing toward the reverse shock, where there is an abrupt decrease in intensity. The reverse shock is much less well defined by $21 \mathrm{~ns}$, as by then the instability has led to modulation [and apparently slowing, by compariperturbations and $100-\mathrm{mg} / \mathrm{cm}^{3}$ foam), Robey et al. evaluated $^{14}$ the kinematic viscosity, concluding that it is approximately $0.05 \mathrm{~cm}^{2} / \mathrm{s}$ in the foam and $0.1 \mathrm{~cm}^{2} / \mathrm{s}$ in the 

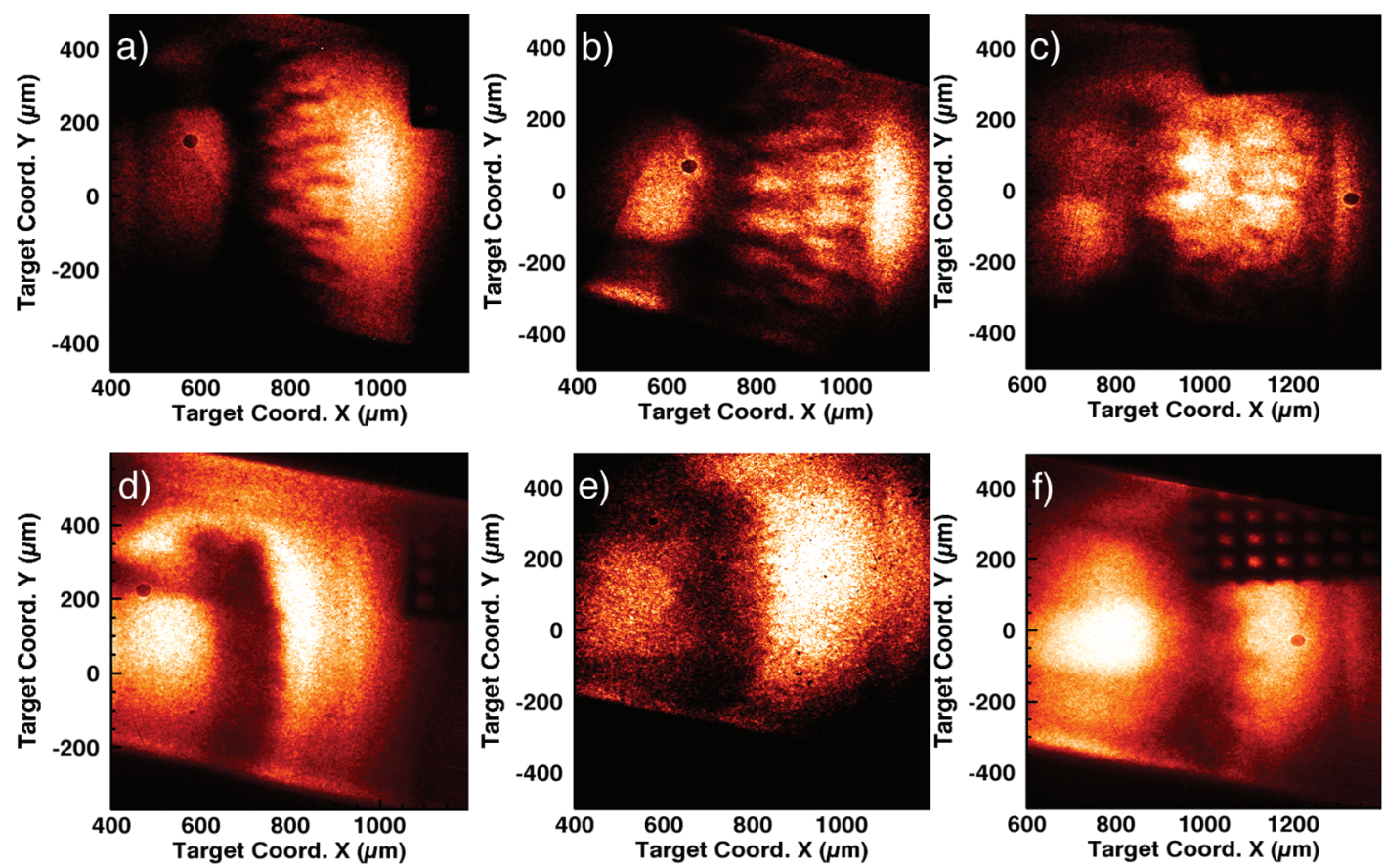

FIG. 5. (Color) Calibrated radiographic data. (a), (b), and (c) show images of modulated targets at 13, 17, and 21 ns, respectively. (d), (e), and (f) show images of planar targets at the same times. These experiments used $50-\mathrm{mg} / \mathrm{cm}^{3}$ foam.

son with Fig. 5(f)] of the entire layer of dense plastic. For the planar targets, the plastic-foam interface is well defined at $940 \mu \mathrm{m}$ in Fig. 5(d), $1110 \mu \mathrm{m}$ in Fig. 5(e), and $1280 \mu \mathrm{m}$ in Fig. 5(f). One does see some evidence of structure that presumably has developed from the intrinsic roughness of the machined surfaces.

For the modulated targets, the images are not simple because of the action of the instability. One can see a series of alternating bright and dark features, which we attribute to the presence of bubbles of foam material interspersed with spikes of CHBr. From these images, it is not so obvious where the left edge of the bubbles (the tips) may be. In fact, the dense plastic material has become modulated through the development of the instability. Thus, a vertical Fourier trans- form of these images shows modulations at the imposed wavelength that extend some distance into the dense material. This may be most evident visually in the image at $17 \mathrm{~ns}$. By $21 \mathrm{~ns}$, the dense material is becoming more globally modulated. One can determine the location of the bubble tips fairly accurately, however, by examining narrow horizontal slices through the image. One sees an abrupt decrease in signal where the foam ends and the $\mathrm{CHBr}$ begins. We used this approach to determine the location of the bubble tips and of the other experimental features for the analysis below.

There is a very structured layer, the "mix layer," extending from the tips of the bubbles to the tips of the spikes, whose details we will discuss further shortly. In the image of Fig. 5(a) at $13 \mathrm{~ns}$, the shock wave in the foam is present
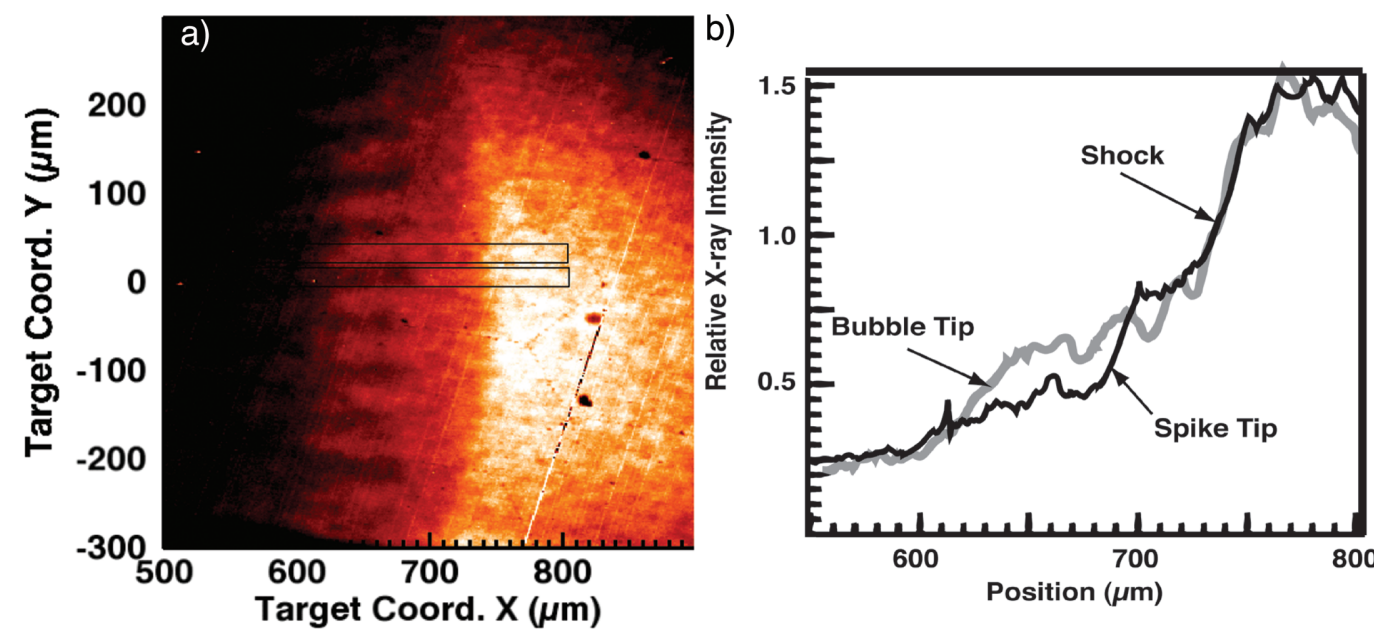

FIG. 6. (Color) Radiographic data and lineouts at $10 \mathrm{~ns}$. (a) The image. The boxes show the lineout locations. (b) Lineouts through a spike and a bubble, showing simple structure in contrast to that of Figs. 5(a) -5 (c). This experiment used $100-\mathrm{mg} / \mathrm{cm}^{3}$ foam. 


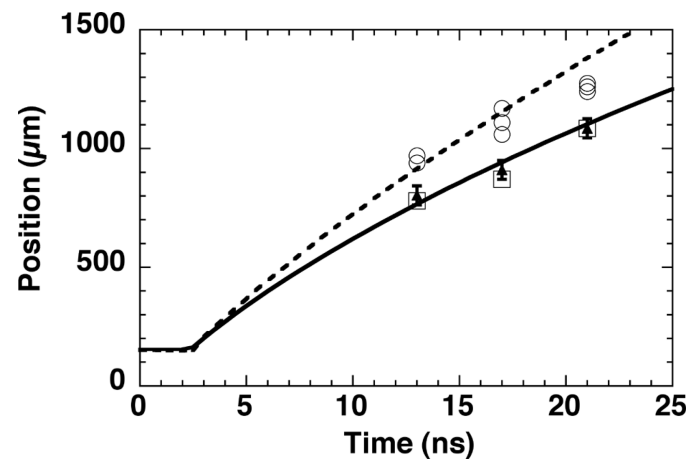

FIG. 7. The simulated evolution of the interface (solid) and shock (dashed) locations is compared with results of measurements of the interface location from the targets with a planar initial interface (open squares), of the shock location (circles), and of the position midway between the bubble tips and spike tips (triangles). Error bars not shown are smaller than the symbols. This figure is for $50-\mathrm{mg} / \mathrm{cm}^{3}$ foam.

about $100 \mu \mathrm{m}$ beyond the end of the spikes, at $970 \mu \mathrm{m}$. This is very difficult to see in the image as displayed; the shockwave location was confirmed in the experiment of Fig. 5(d) with a planar target. By $17 \mathrm{~ns}$, the mix layer has extended to the location of the shock, and this remains the case at $21 \mathrm{~ns}$. Again, this is confirmed by the images of Figs. 5(e) and 5(f), showing that the forward shock in a planar target is located approximately where the spikes end in the modulated target. One caveat here is that the measurement of the shock averages across the entire target. If the shock were pushed forward above the tracer strip one might not detect this, but it also ought to be a small effect.

Now consider the structure in the mix layer. In typical data and simulations of the Rayleigh-Taylor instability in compressible media affected by strong shocks, nearly all of which are either two-dimensional or concerned with the behavior of a single spike, one sees distinct spikes that develop enlarged tips. Indeed, data at $10 \mathrm{~ns}$, shown in Fig. 6, show this same behavior in the present experiments. But this is not what one sees at later times. Instead, moving from left to right in Fig. 5 one sees two sets of spikes that are out of phase with one another. This is present in all three of the images shown here, and may be most evident in image (b) at $17 \mathrm{~ns}$, where there are four particularly transparent bubbles in two pairs that are offset laterally relative to the flow direction. The fact that the spikes overtake the shock is also unusual, and is also discussed further below.

Before interpreting these data, it is useful to quantify the hydrodynamic evolution of this system. Figure 7 shows some aspects of the hydrodynamic evolution, for the $50-\mathrm{mg} / \mathrm{cm}^{3}$ foam data. The solid curve shows the interface position calculated by the simulations. The observed interface motion, in experiments with a planar interface, is consistent with the simulation. The average of the spike and bubble positions also moves with the interface location. This would not necessarily be expected or required; it indicates that in this case the mix layer is roughly symmetric about the original interface position. A dashed line shows the simulated location of the shock wave. The data regarding the shock wave location are from experiments with or without a modulated interface.

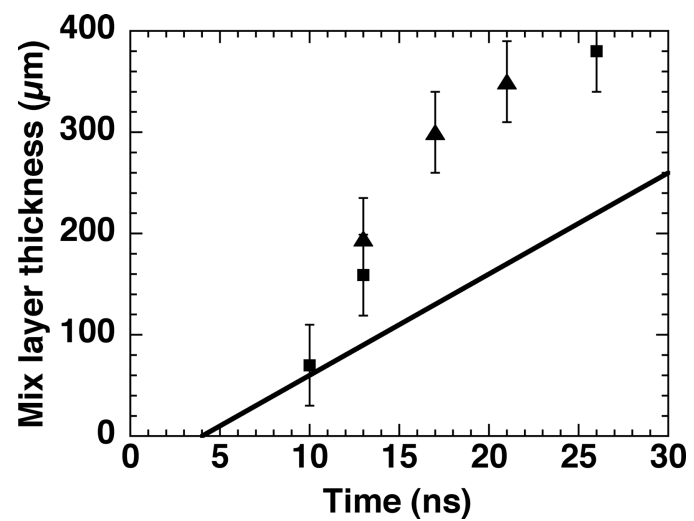

FIG. 8. Growth of the mix layer, defined as the distance between the spike tips and the bubble tips. A line at $10 \mathrm{~km} / \mathrm{s}$ is shown for comparison. The triangles and squares are from $50-\mathrm{mg} / \mathrm{cm}^{3}$ foam and $100-\mathrm{mg} / \mathrm{cm}^{3}$ foam, respectively.

At the earliest time shown (and at $10 \mathrm{~ns}$ for $100-\mathrm{mg} / \mathrm{cm}^{3}$ foam), the data and the simulation agree on the shock location. At $17 \mathrm{~ns}$, the picture is mixed. In one case, the shockwave location lies on the simulation result. In another case, both the shock and the average position of the bubbles and spikes lie below those of the simulation. The laser energy was not anomalously low. We can assess the metrology from photographs, and any error was much smaller than would be needed to make up the observed difference. By $21 \mathrm{~ns}$, the shock wave has slowed significantly in comparison with the value in the simulation. There are least two possible causes of this. On the one hand, the shock becomes curved by this time, and 2D simulations of the entire target reproduce the observed slowing of the shock. ${ }^{15}$ On the other hand, one also cannot rule out the possibility that the foam equation of state may be inaccurately characterized by the tables used.

A second measure of the hydrodynamic behavior is the evolution of the mix layer itself. Figure 8 shows the separation of the bubble tips and spike tips as a function of time in the experiments, with a line at $10 \mathrm{~km} / \mathrm{s}$ shown to provide a scale. The latest data point, at $26 \mathrm{~ns}$, shows the distance between the modulated surface of the dense plastic (taken to be bubble tips or remnants thereof) and the shock, as no intermediate structures are resolved at that time. Recall that the initial amplitude of the perturbation was $5 \mu \mathrm{m}$, large enough (after the shock compression and initial rarefaction) that one would anticipate at most a very limited linearinstability phase with exponential growth. The rate of growth slows after the spikes reach the shock and as the shock slows. The expansion of the mix layer contributes some of the observed growth. The material between the interface and the forward shock has expanded, relative to its immediate post-shock value, by an average of $24 \%$ at $10 \mathrm{~ns}, 32 \%$ at 13 ns, and $48 \%$ at $21 \mathrm{~ns}$.

The following sequence of events is seen in the data. First, there is a period during which the spikes and bubbles become larger. Next, there is a change in the morphology of the mix layer as a second set of spikes, spatially offset relative to the first, forms. Figure 5 implies that this shift in phase occurs approximately at the location of the unper- 
turbed interface. After that, the mix layer grows rapidly and overtakes the shock. [This occurs first in the case of Fig. 5(b), which is unfortunately the experiment with anomalously small shock and interface displacements, but also occurs at $13 \mathrm{~ns}$ for $100-\mathrm{mg} / \mathrm{cm}^{3}$ foam, in an experiment whose shock and interface displacements are consistent with simulations.]

\section{DISCUSSION}

The first observation from the data is that the spike growth seems quite rapid, by comparison with the typical behavior of 2D spikes. This rapid growth of the spikes in the data leads one to ask whether or not their behavior is consistent with the buoyancy-drag models that are often used to describe RT turbulence, and are fairly successful at describing the mix-layer growth for two-dimensional single-mode perturbations. ${ }^{15}$ It makes sense here to examine the growth of the spikes as we can determine this more accurately. In addition, one might hope the model would be more accurate for the spikes, as during their evolution there is little compression and the theory is formally for incompressible fluids. We examined the implications of a standard 3D buoyancy-drag equation for the spikes,

$$
\frac{d u}{d t}=-A g-\frac{(1-A)}{2} k u^{2},
$$

in which $u$ is the time derivative of the distance from the spike tips to the interface, $g$ is the acceleration of the interface (which is negative, as the interface decelerates), and $k$ is the wave number of the perturbation. The Atwood number is designated by $A$ and equals $\left(\rho_{1}-\rho_{2}\right) /\left(\rho_{1}+\rho_{2}\right)$, where $\rho_{1}$ and $\rho_{2}$ are the densities on the higher-density and lowerdensity sides of the interface, respectively. The density drop is about 5. We obtained Eq. (1) from Oron et al., ${ }^{33}$ but similar or identical equations are discussed in numerous papers, reviewed by Dimonte. ${ }^{34} \mathrm{~A}$ fit to the simulation for the $50-\mathrm{mg} / \mathrm{cm}^{3}$ foam gives $A=0.7$ and $g=-6.4(t / 3)^{-1.1}$, while a fit to the simulation results for the $100-\mathrm{mg} / \mathrm{cm}^{3}$ foam gives $A=0.62$ and $g=-7.5(t / 3)^{-1.2}$, with $g$ in $\mu \mathrm{m} / \mathrm{ns}^{2}$ and $t$ in ns. Integrating Eq. (1) for the experimental $k$ produces the results shown in Fig. 9. At $13 \mathrm{~ns}$, the spikes have nearly reached the shock, and have averaged about $3 / 4$ of the velocity of the interface before deceleration, known as the "free-fall" velocity. After 13 ns, the spikes are at the shock and their length is controlled by the shock. The only earlier data point is close to the spike length predicted by the buoyancy-drag model. Though more data from future experiments would solidify this conclusion, the data of Fig. 9 suggest that there may be a brief period of very rapid spike growth from 10 to $13 \mathrm{~ns}$. This is both remarkable and unanticipated. The expansion discussed above would not explain the growth during this interval and is not enough to make up the difference between the model and the data at $13 \mathrm{~ns}$. A second observation in need of explanation is that the spike morphology changes after 10 ns. The data, a twodimensional projection of the spikes in the $\mathrm{CHBr}$ layer, show a shift in the spike location by $1 / 2$ the spike spacing.

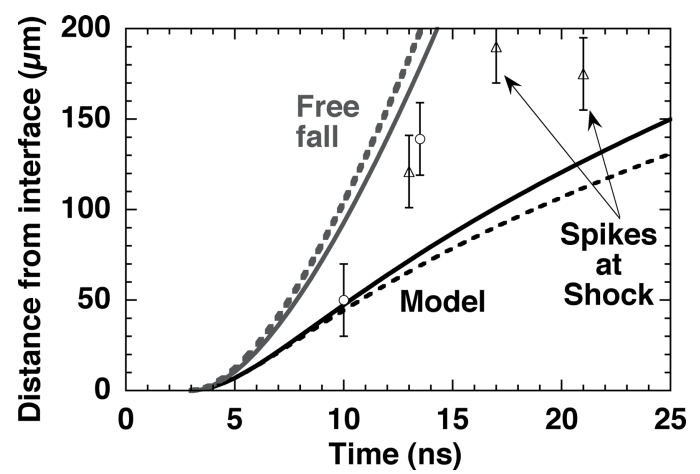

FIG. 9. Spike growth and modeling. The lower curves show the spike length predicted by Eq. (1), while the upper curves show the distance from the free-fall line to the interface. The solid curves are for $50-\mathrm{mg} / \mathrm{cm}^{3}$ foam and the dashed curves are for $100 \mathrm{mg} / \mathrm{cm}^{3}$ foam. The triangles and solid dots show the spike length for $50-\mathrm{mg} / \mathrm{cm}^{3}$ foam, relative to the observed location of the interface for planar targets, and for $100-\mathrm{mg} / \mathrm{cm}^{3}$ foam, relative to the simulated location of the interface, respectively.

We should note that the analytic analyses have been limited to either single bubbles or large multimode statistical ensembles. We did note in the Introduction that the computational analysis is rather limited. The structure seen in the present data clearly does not correspond to the broadband, multimode turbulence that might lead one to see growth of the mix layer depth with time $t$ proportional to $(1 / 2) \alpha g t^{2}$, where $g$ is the acceleration and $\alpha$ is a factor.

Now we consider potential explanations for these observations. Any mechanism that introduces additional vorticity can cause spikes to lengthen, as seen, for example, in the work of Jun and Jones on the interaction of spikes with clumps in supernova remnants. ${ }^{35}$ There is a step at the edge of the $\mathrm{CHBr}$, whose surface may be displaced by as much as $4 \mu \mathrm{m}$ relative to the surface of the polyimide (because of the difference in the response of the two materials to machining). The presence of this step will introduce additional vorticity, and this might differ between alternating rows of spikes. This could cause the spikes to penetrate to the shock and could produce the difference in the absorption of alternating rows of spikes seen in some images. However, it is not clear how this could produce very transparent bubbles lying directly below the spikes as in Fig. 5(b).

The morphological changes and the increased spike penetration might also arise through the interaction of the spike tips As the initial spike tips broaden, their ability to interact also increases. Suppose they interact so as to direct the flow of matter into new spikes. This might perhaps lead to an initial period of rapid growth in the second tier of spikes. One can imagine that this process could repeat itself again if the spikes had not overtaken the shock. (The increased drag on spikes that penetrate beyond the shock effectively prevents the spikes from emerging ahead of it.) Such interactions might potentially explain the observation of bubbles "under" the spikes (in the flow direction), as is observed, but the simplest interactions along symmetry axes would keep the spikes aligned in the diagnostic view, assuming perfect target construction and alignment and planar flow.

There is some support for the possibility that the spike 
tips may interact in the $2 \mathrm{D}$ simulation work recently reported $^{36}$ by Miles et al. In this work, the interaction of spike tips leads to the production of jets of denser material that penetrate further into the low-density material. The simulations include two modes, with the initial wavelength and amplitude of the second mode being 1/10 those of the first mode. These authors attribute the formation of the jets to the interaction of the two modes, and do not see them when the second mode is not present. In the present experiments, the high-frequency modulations of the interface seen in Fig. 3 may play the role of enabling the formation of secondary spikes. On the other hand, in 3D such additional modulations might not be required.

There might be some other explanation for both the morphology and the spike penetration, but we have been unable to find a credible one. Certainly a second shock might produce such effects, but there is no source for one in this open experimental geometry with only a single, brief, period of energy input. (Note that the initial shock is only halfway through the dense plastic when the laser pulse ends.) Larger, faster-growing structures might be produced through the merger of the bubbles, but it would seem that this ought to be more apparent in the image data. The preheat that might be present could cause evolution of the initial interface before the shock arrived. Even unrealistically high levels of preheat, however, produce only some rarefaction at the interface and do not produce a shock through the interface. While this potentially could spoil the ability of simulations to reproduce the data, it would not alter the qualitative evolution of the system, beginning from a long-wavelength perturbation with additional, shorter-wavelength modulations.

Finally, there is no reason to believe that the flow of material up the spikes will cease once the spikes reach the shock. We speculate that under some circumstances the 3D Rayleigh-Taylor instability may be able to deliver significant amounts of material to the vicinity of the forward shock. In the present case, quantitative assessment of this possibility is not feasible in view of the rapidly changing intensity of the backlighter $\mathrm{x}$ rays across this portion of the images. This could produce much more rapid transport of material in laboratory and astrophysical systems than one would otherwise expect. The implications of this could be dramatic, but their exploration is beyond the scope of the present paper.

\section{FUTURE DIRECTIONS}

In summary, we have studied a system in which the Rayleigh-Taylor instability produces the long-term growth of structures at a decelerating interface produced when a blast wave encounters a density drop. We created 3D initial conditions such that spike material is propelled toward the transmitted shock at a rate faster than is easily explained. The results discussed here and their apparent implications are sufficiently novel that careful follow-up studies are warranted to determine what really happens to three-dimensional Rayleigh-Taylor instabilities in compressible, strong-shockinitiated conditions. Experimentally, the measurements are definitely limited by both noise and contrast, so the development of improved diagnostic techniques, such as backlit pin- hole measurements or curved-crystal imagers, would be of value, as would the development of higher-contrast tracer layers, such as iodine-doped plastic. Using either a dense gas or cryogenic hydrogen would also help, by removing some of the uncertainties associated with the foam equation of state. It would be useful as well to obtain experiments of longer duration, but this would require producing highervelocity blast waves. This might become feasible on the National Ignition Facility, but perhaps at the expense of complications from hot-electron preheat.

Computationally, the results motivate the use of the new generation of fast, adaptive hydrodynamic codes to evaluate 3D Rayleigh-Taylor behavior in compressible, strongly shocked systems. A systematic elucidation of the regimes of spike interactions remains to be attempted, and would provide valuable guidance to the interpretation of phenomena observed both in the laboratory and throughout the universe.

\section{ACKNOWLEDGMENTS}

We acknowledge useful discussions with Dmitri Ryutov and useful technical contributions Mike Grosskopf, Amy Reighard, Leah Norstrund, and Kelly Korreck. We acknowledge the technical support of the target fabrication personnel at the Lawrence Livermore National Laboratory, and of the Omega Laser Operations team. Both were essential to the success of this work.

Financial support for this work included funding from the U.S. Department of Energy to the University of Michigan under Grants No. DE-FG03-99DP00284 and No. DE-FG0300SF22021, and to the Lawrence Livermore National Laboratory under Contract No. W-7405-ENG48.

${ }^{1}$ L. Rayleigh, Scientific Papers II (Cambridge University Press, Cambridge, England, 1900).

${ }^{2}$ S.G. Taylor, Proc. R. Soc. London, Ser. A 201, 192 (1950).

${ }^{3}$ J.D. Kilkenny, S.G. Glendinning, S.W. Haan et al., Phys. Plasmas 1, 1379 (1994).

${ }^{4}$ C.J. Pawley, S.E. Bodner, J.P. Dahlburg et al., Phys. Plasmas 6, 565 (1999).

${ }^{5}$ J.P. Knauer, R. Betti, D.K. Bradley et al., Phys. Plasmas 7, 338 (2000).

${ }^{6}$ K.S. Budil, B.A. Remington, T.A. Peyser et al., Phys. Rev. Lett. 76, 4536 (1996).

${ }^{7}$ J. Kane, D. Arnett, B.A. Remington et al., Astrophys. J. Lett. 478, L75 (1997).

${ }^{8}$ J. Kane, D. Arnett, B.A. Remington et al., in Second Oak Ridge Symposium on Atomic and Nuclear Astrophysics, Oak Ridge, Tennessee, 1998.

${ }^{9}$ J. Kane, D. Arnett, B.A. Remington, et al., Phys. Plasmas 6, 2065 (1999).

${ }^{10}$ J. Kane, D. Arnett, B.A. Remington et al., Astrophys. J., Suppl. 127, 365 (2000).

${ }^{11}$ J.O. Kane, H.F. Robey, B.A. Remington et al., Phys. Rev. E 63, 055401(R) (2001).

${ }^{12}$ H.F. Robey, J.O. Kane, B.A. Remington et al., Phys. Plasmas 8, 2446 (2001).

${ }^{13}$ R.P. Drake, H.F. Robey, O.A. Hurricane et al., Astrophys. J. 564, 896 (2002).

${ }^{14}$ H.F. Robey, Y. Zhou, A.C. Buckingham et al., Phys. Plasmas 10, 614 (2003).

${ }^{15}$ A.R. Miles, D.G. Braun, M.J. Edwards et al., "Numerical simulation of supernova-relevant laser-driven hydrodynamics experiments on Omega," Phys. Plasmas (to be published).

${ }^{16}$ K. Kifonidis, T. Plewa, H.-T. Janka, and E. Muller, Astrophys. J. Lett. 531, L123 (2000).

${ }^{17}$ B. Fryxell, E. Muller, and D. Arnett, Astrophys. J. 367, 619 (1991).

${ }^{18}$ G. Tryggvason and S.O. Unverdi, Phys. Fluids A 2, 656 (1990). 
${ }^{19}$ J.P. Dahlburg, J.H. Gardner, G.D. Doolen, and S.W. Haan, Phys. Fluids B 5, 571 (1993).

${ }^{20}$ J. Kane, D. Arnett, B.A. Remington et al., Astrophys. J. 528, 989 (2000).

${ }^{21}$ J. Hecht, D. Ofer, U. Alon et al., Laser Part. Beams 13, 423 (1995).

${ }^{22}$ J.P. Dahlburg, D.E. Fyfe, J.H. Gardner, S.W. Haan, and G.D. Doolen, Phys. Plasmas 2, 2453 (1995).

${ }^{23}$ Y.-N. Young, H. Tufo, A. Dubey, and R. Rosner, J. Fluid Mech. 447, 377 (2001).

${ }^{24}$ D.L. Youngs, Phys. Fluids A 3, 1312 (1991).

${ }^{25}$ D. Shvarts, U. Alon, D. Ofer, R.L. McCroy, and C.P. Verdon, Proceedings of the Fifth International Workshop on Compressible Tubulent Mixing (1995), p. 66.

${ }^{26}$ M.M. Marinak, B.A. Remington, S.V. Weber et al., Phys. Rev. Lett. 75, 3677 (1995).

${ }^{27}$ M.M. Marinak, S.G. Glendinning, R.J. Wallace et al., Phys. Rev. Lett. 80, 4426 (1998).

${ }^{28}$ G. Dimonte and M. Schneider, Phys. Rev. E 54, 3740 (1996).
${ }^{29}$ T.R. Boehly, R.S. Craxton, T.H. Hinterman et al., Rev. Sci. Instrum. 66, 508 (1995).

${ }^{30}$ K.S. Budil, T.S. Perry, P.M. Bell et al., Rev. Sci. Instrum. 67, 485 (1996).

${ }^{31}$ J.T. Larsen and S.M. Lane, J. Quant. Spectrosc. Radiat. Transf. 51, 179 (1994).

${ }^{32}$ L.D. Landau and E.M. Lifshitz, Fluid Mechanics (Pergamon, Oxford, 1987).

${ }^{33}$ D. Oron, L. Arazi, D. Kartoon et al., Phys. Plasmas 8, 2883 (2001).

${ }^{34}$ G. Dimonte, Phys. Plasmas 7, 2255 (2000).

${ }^{35}$ B.I. Jun, T.W. Jones, and M.L. Norman, Astrophys. J. Lett. 468, L59 (1996).

${ }^{36}$ A.R. Miles, M.J. Edwards, and H.F. Robey, "The effect of a shortwavelength mode on the nonlinear evolution of a long-wavelength perturbation driven by a strong blast wave," Proceedings of the Third International Conference on Inertial Fusion Sciences and Applications, September 2003 (in press). 